\title{
Investigating Malware Campaigns with Semantic Technologies
}

\author{
Rodrigo Carvalho*†, Michael Goldsmith*, Sadie Creese* \\ *Department of Computer Science \\ University of Oxford, Oxford, UK \\ \{rodrigo.carvalho, michael.goldsmith, sadie.creese\}@cs.ox.ac.uk \\ †Brazilian Federal Police, Brasília, Brazil \\ carvalho.rac@dpf.gov.br
}

\begin{abstract}
Malware-campaign investigation is a major factor in fighting cybercrime. Most of the research in this area comes from commercial companies, so potentially there is a greater emphasis on detection rather than malware attribution. Aiming at a better balance between human reasoning skills and computer processing capabilities, our hypothesis is that semantic technologies could greatly enhance the investigation of the malware ecosystem.

To demonstrate this, we have reproduced using our prototype the investigation rationale described in a report from 2015 . The transparent methodology regarding the analysis of 52 files allowed us to translate the domain knowledge of the authors of the report into entities, relationships and rules. As an extension to the case study, we have further enriched our knowledge base with 155 new files. This led to the prompt identification of relationships and patterns among the new dataset entities in a semi-automated, scalable fashion, confirming our hypothesis.
\end{abstract}

\section{MALWARE CYBERCRIME INVESTIGATION}

Cybercrime is a major problem which is directly associated with the current high level of connectivity and data ubiquity within our society. Surveys from organizations such as Anti-Phishing Working Group(APWG) [1] inform about its alarming rates:

- "The total number of unique phishing" sites observed in the second quarter of 2016 was 466,065. This was an all-time high."

- "APWG member PandaLabs found 18 million new malware samples in Q2, an average of more than 200,000 a day. This is 10 percent lower than in the previous quarter, when 20 million new samples were found."

One of the proposed reasons for such widespread cybercrime is that there are many places on the Internet in which cybercriminals trade malware and infrastructure

1. Phishing is the attempt to acquire sensitive information such as usernames, passwords, and credit card details (and sometimes, indirectly, money), often for malicious reasons, by masquerading as a trustworthy entity in an electronic communication. Source: https://en.wikipedia.org/wiki/Phishing, accessed in 26/11/2016. services, such as botnet $^{2}$ rental, at competitive prices, see [2].

AV vendors, who form a large part of the security community, tend to tackle this problem by detecting and black-listing the malicious files and web servers distributing them. This makes sense since their main commercial focus is securing the computers of their clients, and not investigating the associated malware campaigns.

Determining the attribution of a malware campaign is typically the responsibility of Law Enforcement Agencies (LEAs) because it is a criminal act. However, possibly due to the difficulty of such task, most agencies focus on disrupting the computer infrastructure used to spread malware. Operation Avalanche [3] is a one of the most recent documented cases.

Although demanding a major cooperative effort spanning several jurisdictions, disruption is an effective strategy to stop malware distribution at large scale, ultimately leading to increasing the costs of cybercrime activity. However, criminals on the loose can always hire different infrastructure and acquire upgraded malware, in a continuous "cat and mouse" game. We believe that more productive investigations could not only bring them to justice but also act as a deterrent.

One of the most challenging issues in investigating a malware campaign is producing comprehensive evidence against its perpetrators. Often, probative and courtadmissible evidence can only be found after seizing the devices owned by the criminal organizations. Obtaining such devices is a complex task, even if they are within the same jurisdiction of the LEA: investigators must hypothesize about a high volume of heterogeneous, supposedly

2. A botnet is an interconnected network of computers infected with malware without the user's knowledge and controlled by cybercriminals. They're typically used to send spam emails, transmit viruses and engage in other acts of cybercrime. Source: https://usa.kaspersky.com/internet-security-center/threats/botnetattacks, accessed in 27/04/2016. 
unrelated Indicators of Compromise ${ }^{3}$ (IOCs) for patterns and relationships which could eventually support a search warrant.

More than ever, "Todays tools must be re-imagined to facilitate investigation and exploration" [4]. We address this particular challenge with a prototype that leverages semantic technologies to integrate such horizontally-spread data and facilitate the insight-building process of the analysts. The purpose of our research being to explore and demonstrate the utility of semantic technologies in this domain.

\section{Semantic technologies}

It is probably easier to explain what semantic technologies are if we present the idea behind the Semantic Web as defined by Tim Berners-Lee: "The Semantic Web is not a separate Web but an extension of the current one in which information is given well-defined meaning, better enabling computers and people to work in cooperation" [5].

Such well-defined meaning is given to information through an ontology, which aims to explicitily define meanings shared by a community [6]. The basic components of an ontology are:

- Classes: describe concepts, or the type of things within a domain. In our case study, Webserver and File are two classes, and PayloadFile is a subclass of the latter, inheriting all its properties.

- Properties: relationships between members of different classes (object properties) or between members of a class and a literal (data properties). Some examples would be PayloadFile connectsTo WebServer and File name string, respectively.

- Individuals: the instances of a class (or the objects described by it). For instance, evil.org could be one instance of the class WebServer. Often, the Individuals are not considered part of the ontology per se, but together with it form what is called a knowledge base.

Classes, properties, individuals and all the other components of a knowledge base are logically stored as triples (subject predicate object), describing one statement each. Triples are represented using the Resource Description Framework ${ }^{4}(\mathrm{RDF})$. RDF uses web-based URIs to name the relationship between things as well the things themselves, allowing structured and semi-structured data to be integrated and shared across different applications.

Listing 1 presents the components of a sample ontology as triples in Turtle syntax. Turtle is a RDF serialization which is compact, easy to read by humans and also a W3C recommendation:

3. Indicator of compromise (IOC) in computer forensics is an artifact observed on a network or in an operating system that with high confidence indicates a computer intrusion. Source: https://en.wikipedia.org/wiki/Indicator_of_compromise, accessed in 07/05/2017.

4. RDF is a standard model for data interchange on the Web. RDF has features that facilitate data merging even if the underlying schemas differ, and it specifically supports the evolution of schemas over time without requiring all the data consumers to be changed. Source: https://www.w3.org/RDF, accessed in 07/05/2017
Listing 1: Ontology definition in Turtle syntax.

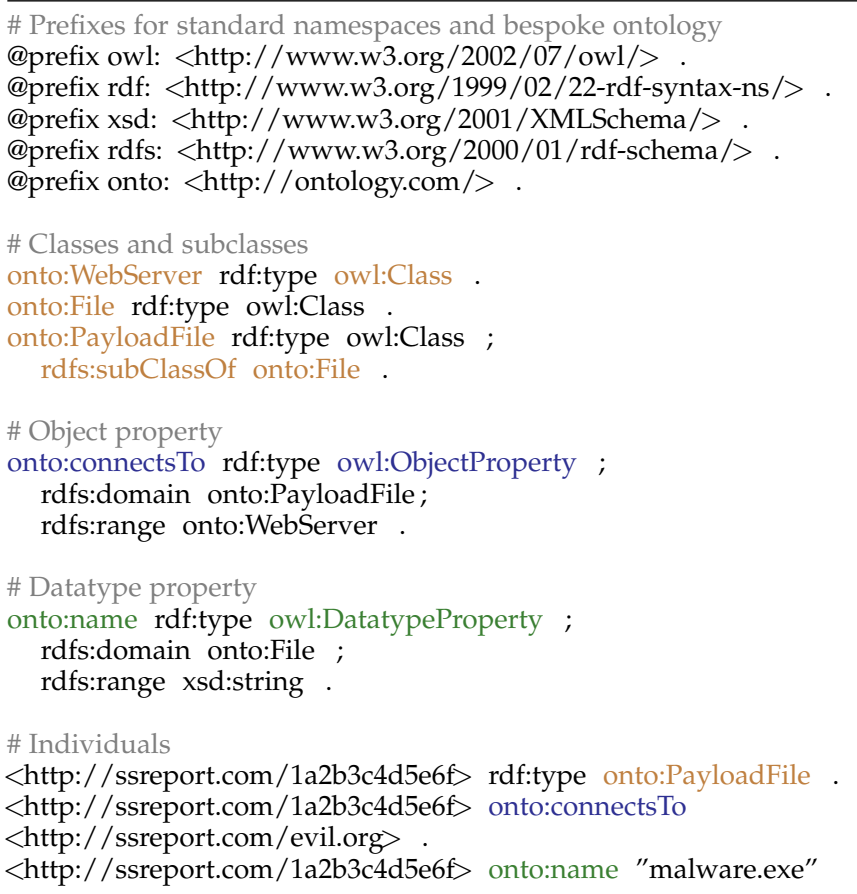

As Listing 1 illustrates (through the PREFIX clauses), one of the key characteristics of the Semantic Web is that classes and properties are defined by Uniform Resource Identifiers (URIs). This ensures that they have an unique definition accessible by anyone on the Web, avoiding concept misunderstanding across different contexts [6].

For instance, one web-master could mark-up the universities described in her site using the definition present in https://schema.org/Organization. This would enable any software agent aware of schema.org (e.g. a search engine) to recognize "University of Oxford" as an organization, which would then be a "thing" instead of a string. Consequently, this "thing" could now hold the relationship https://schema.org/employee with multiple individuals marked-up as https://schema.org/Person.

Of course, "The computer doesn't truly 'understand' any of this information, but it can now manipulate the terms much more effectively in ways that are useful and meaningful to the human user." [5]. A very good example is linking disparate resources describing the same "thing", which could be very useful for data enrichment while keeping its provenance. This will be further discussed in Section 5.4 below.

\section{THE INVESTIGATION OF MALWARE CAMPAIGNS}

Our objective is not to suggest an ontology for malware investigation in the terms of the aforementioned schema.org. There are already comprehensives taxonomies whose concepts could be used to define one, such as STIX ${ }^{5}$, OpenIoc ${ }^{6}$ and MISP $^{7}$. Their development is a consequence of the

5. Structured Threat Information Expression. https://oasisopen.github.io/cti-documentation

6. https://github.com/mandiant/OpenIOC_1.1

7. Malware Information Sharing Platform https://github.com/MISP/misp-objects 
modern understanding that cyber-threat intelligence cannot be effectively represented as an uncontextualized flat list of IOCs, as it describes complex and richly connected entities [7].

However, it is debatable whether current threatintelligence platforms, whether leveraging such taxonomies or processing raw IOCs, are actually producing intelligence. Most of them still focus on data collection rather than analysis, which is often limited to searching, browsing and attribute-filtering [8] as opposed to knowledge and intelligence creation.

Thus, we consider our approach a step towards completing the intelligence cycle, as it aims to facilitate the insight-building process of the investigator during the analysis stage. For that, we believe semantic technologies could excel in the following tasks:

- Easy data integration across disparate data sources: because it might be infeasible for all stakeholders to adopt a particular schema, a straightforward way to define mappings between them is recommended;

- Facet querying: in addition to interactive filtering, it helps understanding the relevant dimensions of a particular domain [9];

- Provenance, or efficiently tracing back any piece of information to the original data source.

Szekely et al describe how such tasks have improved the investigation of human trafficking in a law-enforcement setting [10]. We agree but would go further as we consider that other native capabilities could further enhance relationship-searching and hypothesis-testing within the malware-campaign investigation domain, as detailed in Table 1 .

TABLE 1: Mapping needs of an investigation to compatible semantic capabilities.

Common investigation needs Clustering entities

Establishing links

Inserting tags or comments

Merging data about entities

Reproducing investigation steps

6 Rolling back in case of dead end

Features 1 to 4 are mostly used for building knowledge bases before the users of linked-data exploratory systems can browse and search them. This holds true for most systems described in [9] which, despite sharing some similar objectives with our prototype, do so using different techniques. For instance, using in-session memory to memorize the user browsing sequences and suggesting queries to inspire exploration.

The novelty of our approach lies in actually allowing the user to manipulate these features in order to shape knowledge which would inform his investigation hypotheses. In addition, we believe Feature 5 could be useful for automation and reproducibility purposes, and Feature 6 to avoid repeating prior investigative steps. In order to implement these semantic capabilities, a Triple Store is necessary.

\section{Triple Stores and RDFox}

Triple Stores (or RDF Stores) are a kind of graph database with the added benefits of knowledge-inferencing and materialisation using rules and set processing. Graph databases are more suited to store and query highlyconnected data than relational database systems, facilitating analysis tasks in which relationships and connection patterns between different items is important, see [11] for discussion.

Although native graph databases outperform Triple Stores in data-processing performance, the latter are better suited for information-modelling and sharing. Moreover, their inherent capability of integrating datasets is relevant, as current threat-intelligence platforms provide limited automated data-integration support, see [8] for explanation.

We consider that leveraging a graph technology which favours data integration contributes to investigation pivoting, described by [7] as the fundamental analytic task of hypothesis-testing which relies on the ability of analysts to understand the relationship between elements from distinct data sources. Therefore, we have adopted Triple Stores.

In order to assess the benefits and the feasibility of the ideas discussed in this section, we have implemented the capabilities from Table 1 in a prototype that leverages RDFox, which is a main-memory Triple Store coupled with a parallel datalog reasoner ${ }^{8}$, from the University of Oxford. In addition to supporting sparql (a sql-like query language for RDF, based in graph pattern matching and endorsed by W3C) and handling the "owl:sameAs" object property efficiently, RDFox is very fast at computing and updating data materialisations [12]. The importance of these features to our approach will become clear in Section 5, which reproduces the investigation presented in the next section.

\section{THE Italian Connection REPORT}

A significant cyber-security event happened in 2015, in which data from the company Hacking Team was leaked to the internet. Among the exposed files, there were some zero-day vulnerabilities which were promptly harnessed by other hackers.

Following this breach, researchers at Shadow Server Foundation published a report [13] aiming to revealing relationships between supposedly independent groups based on similarities across IOCs such as payload ${ }^{9}$ and command and control infrastructure. This report will henceforth be referred as $I t C o$, in reference to its original name "The Italian Connection".

Differently from most reports describing malware campaigns, the ItCo report clearly states the methodology used (which investigative step was taken at each stage, and why), explains the rationale behind each assertion they make (e.g.

8. A semantic reasoner, reasoning engine, rules engine, or simply a reasoner, is a piece of software able to infer logical consequences from a set of asserted facts or axioms. The notion of a semantic reasoner generalizes that of an inference engine, by providing a richer set of mechanisms to work with. The inference rules are commonly specified by means of an ontology language, and often a description logic language. Source: https://en.wikipedia.org/wiki/Semantic_reasoner, accessed in 07/05/2017.

9. In computer security, payload refers to the part of malware which performs a malicious action. Source https://en.wikipedia.org/wiki/Payload_(computing), accessed in 25/04/2016. 
"We define independent operators as actors that maintain distinct infrastructure without any technical overlaps such as ip history.") and concludes by presenting competing hypotheses regarding whether different exploits might have a unique origin.

The dataset used by the authors of the ItCo report comprises a total of 52 Adobe Flash files exploiting either $\mathrm{CVE}^{10}$ 2015-5119 or CVE-2015-5122, which are the vulnerabilities related to Hacking Team leaks. These samples were obtained by crawling websites known for distributing malware and also searching online repositories such as VirusTotal and Shadow Server. For each sample, IOCs such as domains, ips, hashes and compression method were extracted by static and dynamic sandbox ${ }^{11}$ analysis.

The first step of the investigation was to distinguish the exploit files produced by a common generator tool ${ }^{12}$ from the ones obtained via source-code sharing. According to the authors' knowledge, exploit files produced by the same generator tool share specific features. For instance, they used the following values to define one of the clusters:

- $\quad$ Created on the same date of 7/7/2015;

- $\quad$ Targeted the same vulnerability of CVE-2015-5119;

- Compressed via the LZMA algorithm;

- Contained an embedded payload;

- Had identical ActionScript classes.

All exploits produced by one generator tool would necessarily have all embedded Action Script classes identical. However, some other exploits, despite sharing most features with each other, would have one or more differing classes. In these cases, the authors of the ItCo report would classify such exploits as source-code sharing.

The report does not mention if an automated method was used to compare the classes from each file. Our approach was to first automatically extract all the classes from each file and produce their MD5 hashes. Then, we calculated the Jaccard Similarity Score (JSS) for every two files, using their internal classes MD5 hashes as the set of features. The pairs with a score of 1 were attributed the relationship has JSS-1.0-with, and those with a score between 0.9 and 0.99, has JSS-0.9-with.

By assessing the different clusters created (two samegenerator clusters and three shared source-code ones) and which of their members would potentially have a unique origin (i.e. the same actor), the authors established competing hypotheses about the exploits supply-chain. These were mostly related to the malware-development skills of distinct actors, which groups would be "collaborating" among themselves and the speed with which their exploits were deployed into the wild.

10. CVE is a dictionary of publicly known information security vulnerabilities and exposures. Source: https://cve.mitre.org/, accessed in $27 / 04 / 206$.

11. A sandbox is a security mechanism for separating running programs. It is often used to execute untested code, or untrusted programs. Source: https:// en.wikipedia.org/wiki/Sandbox_(computer_security), accessed in 27/04/2016.

12. Generator tools, or exploit kits, enable "...an operator to quickly and easy bind a payload or remote download url to shellcode in the flash exploit file via a handful of mouse clicks or a simple command." [13]
We consider the ItCo report an excellent opportunity to validate our approach, presented before in a position paper [14]. At that time it was difficult to implement due to the lack of specific domain knowledge (i.e., the rationale used by malware investigators) and the difficulty of obtaining suitable datasets for the case study (now made available by the authors of the report as a spreadsheet containing all the IOCs).

The following sections will demonstrate how we have fully reproduced the ItCo report by harnessing the capabilities mentioned in Table 1 in our prototype.

\section{Semantic InVEstigation}

First, it is necessary to define our ontology, which will be the basis for converting data to a linked format. We can either extend the concepts from an existing ontology or create a new one.

We decided to create a new ontology for two reasons:

- There is not a widely accepted ontology for malware investigation, but only taxonomies which do not follow semantic-technology standards;

- To demonstrate that designing a basic ontology with a few classes and properties, using an editor such as Protege ${ }^{13}$, is not a difficult task.

The datatype properties of our ontology derived from the column headers of the IOCs spreadsheet are shown in Table 2. The first 4 rows also represent classes, as we have chosen md5 and domain to compose the URI of the entities File and Webserver, respectively.

\section{TABLE 2: Mapping IOCs to semantic concepts.}

\begin{tabular}{|l|l|l|}
\hline Column headers & Classes and datatype properties & Sample values \\
\hline swf md5 & File (Exploit File) and md5 & e33cf5b9f...71f4380dd7eb1 \\
\hline payload md5 & File (Payload File) and md5 & 5a22e5aee...77f1351265a00 \\
\hline exploit site & WebServer (ExploitServer) and domain & news.turkceil.tk/movie.swf \\
\hline C2 & WebServer (C2Server) and domain & amxil.opmuert.org \\
\hline payload in swf & embeddedPayload & yes \\
\hline create date of swf & createDate & $7 / 14 / 15$ \\
\hline swf cve & cve & CVE-2015-5122 \\
\hline swf compression & compression & lzma \\
\hline payload family & family & PlugX \\
\hline
\end{tabular}

Figure 1 illustrates the resulting ontology in vow $l^{14}$ notation: circles are classes and green rectangles are datatype properties. The blue rectangles represent object properties, or bespoke relationships between classes reflecting the knowledge disclosed by the authors in the report. Note there are no individuals yet.

Once the ontology is ready, it is necessary to map the data (in our case, the information from the IOCs spreadsheet) onto it. This was accomplished using the tool Karma ${ }^{15}$, which provides an intuitive interface to create the mapping model and later allows for batch processing.

The resulting linked data resembles the individuals depicted in Listing 1: ssreport.com is the bespoke namespace we have defined for our data, and vtinv is the ontology comprising, among others, the property connects To. $1 a 2 b 3 c 4 d 5 e 6 f$ and evil.org are the identifiers for one $\mathrm{F} i \mathrm{le}$ and one Webserver within our namespace, respectively.

13. http:/ / protege.stanford.edu

14. http://vowl.visualdataweb.org

15. http://usc-isi-i2.github.io/karma 


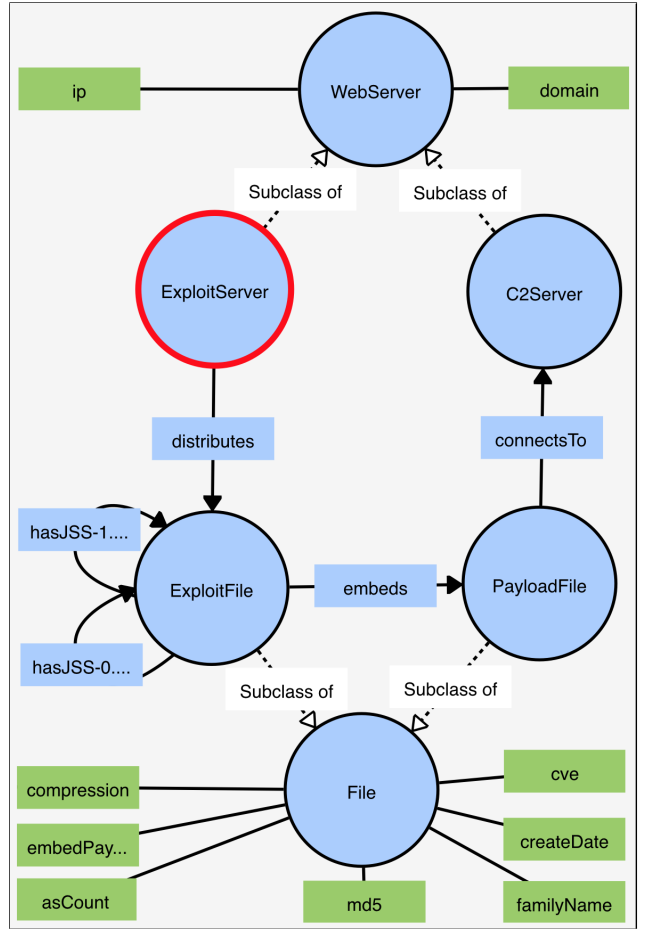

Fig. 1: The vtinv ontology.

Finally, the knowledge base (comprising both the ontology and the data) was loaded into our prototype. The initial number of individuals is shown in Table 3 , column 0.

TABLE 3: Evolution of the number of individuals. Bold denotes a change in value, and ' - ' means the concept is not present in that iteration.

\begin{tabular}{l|llllllll} 
Iteration & 0 & 1 & 2 & 3 & 4 & 5 & 6 & 7 \\
\hline File & 74 & $\mathbf{4 3}$ & 43 & $\mathbf{7 0}$ & $\mathbf{4 3}$ & $\mathbf{1 9 8}$ & $\mathbf{1 6 1}$ & $\mathbf{1 3 7}$ \\
new_HT & - & $\mathbf{1 1}$ & 11 & 11 & 11 & 11 & 11 & $\mathbf{3 1}$ \\
new_002 & - & $\mathbf{1 2}$ & 12 & 12 & 12 & 12 & 12 & 12 \\
new_exp1 & - & $\mathbf{6}$ & 6 & 6 & 6 & 6 & 6 & $\mathbf{1 0}$ \\
new_exp2 & - & $\mathbf{2}$ & 2 & 2 & 2 & 2 & 2 & 2 \\
WebServer & 65 & 65 & 65 & $\mathbf{8 1}$ & $\mathbf{7 1}$ & $\mathbf{1 0 9}$ & $\mathbf{1 0 3}$ & 103 \\
hasJSS-1.0 & 230 & 230 & 230 & 230 & 230 & $\mathbf{1 1 7 4}$ & $\mathbf{9 7 0}$ & 970 \\
hasJSS-0.9 & 150 & 150 & 150 & 150 & 150 & 150 & 150 & 150 \\
connects & 42 & 42 & 42 & $\mathbf{6 2}$ & $\mathbf{5 0}$ & $\mathbf{9 6}$ & $\mathbf{9 1}$ & 91 \\
distributes & 36 & 36 & 36 & 36 & 36 & 36 & 36 & 36 \\
embeds & 37 & 37 & 37 & 37 & 37 & $\mathbf{7 3}$ & 73 & 73 \\
sameC2as & - & - & $\mathbf{1 4}$ & 14 & 14 & 14 & 14 & $\mathbf{3 8}$ \\
sameC2ESas & - & - & $\mathbf{2}$ & - & - & - & - & -
\end{tabular}

The number of Files in each iteration includes both Exploitfiles and their embedded PayloadFiles (obtained from the columns swf md5 and payload md5 of the IOCs spreadsheet). Therefore, there is no conflict with the initial count of exploit files given in Section 4 (52).

\subsection{Semantic Facet querying}

As mentioned before, the initial step of the authors of the ItCo report was to cluster files potentially created by the generator. After exploring the data, they come to the cluster definition given in Section 4.

Although the report does not mention their exploratory process, we could simulate it using facet querying: we firstly queried for Files which hold the relationship hasJSS-1.0-with with another File. Analysing the results it was clear to notice that Compression and CVE would be good features for clustering.

This is a basic use of the facet querying, and the fact that we are analysing previously processed data certainly made it easier to spot good cluster features. Hence, a more complex query will be demonstrated below in Subsection 5.3, and Subsection 5.4 will demonstrate our approach within a larger dataset.

Our prototype allows for semantic facet queries to be built in a systematic fashion. Figure 2 illustrates the query builder interface, in which the content of the drop-down boxes is updated according to the results of the previous run. It is possible to incrementally append relationships between instances (first column) and restrictions to datatype properties using either existing literals (second column) or logical and numerical operators (third column).

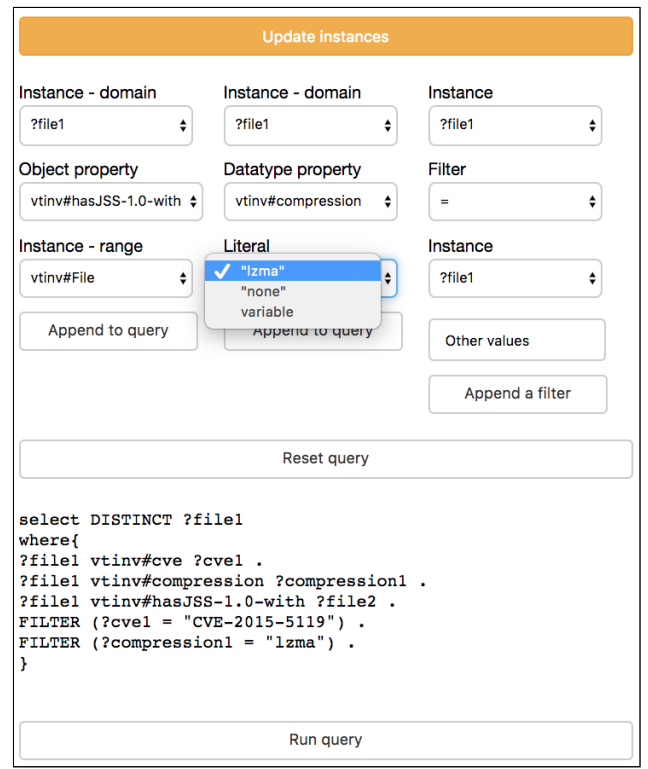

Fig. 2: The query builder. Items with "?" represent placeholders for distinct class instances, and items in quotes are literals.

Running the query from Figure 2 on the knowledge base yielded the same results as Table 1 from the ItCo report (which enumerates the members of the HT_exploit cluster). If deemed relevant for the investigation, the individuals returned by the query can easily be grouped into a new class.

\subsection{Clustering or tagging entities}

The similar syntax of sparql queries and the datalog rules expected by RDFox makes it easy to transform the previous query into a class definition. The rule depicted in Listing 2 is automatically generated by our prototype, and could be applied any time new data is loaded into the knowledge base.

Listing 2: Rule derived from the sparql query in Figure 2. vtinv:new_HT(?file1) :- 
vtinv:compression(?file1, ?compression1),

vtinv:hasJSS-1.0-with(?file1, ?file2),

vtinv:cve(?file1, ?cve1),

FILTER (?cve1 = "CVE-2015-5119"),

FILTER (?compression1 = "lzma")

Thereafter, it is necessary to materialize the new facts resulting from this rule into our knowledge base. This is accomplished by invoking the reasoner, and would result in a new class vtinv:new_HT, subclass of vtinv:File, containing all individuals matching the rule.

Likewise, we could simply tag the individuals returned by the previous search. Suppose we want to attribute a confidence level regarding their origin. Then, instead of creating a new class, we could define the datatype property confidence_generator with the value high. To accomplish that, it would be enough to update the first line of Listing 2 to:

Listing 3: Defining or updating a datatype property.

vtinv:confidence_generator(?file1, 'high') :-

We believe that this feature, in addition to the native property rdfs : comments, could also be useful for easily documenting and retrieving non-technical information, such as the Tactics, Techniques, and Procedures (TTPs) of the cybercriminals.

Going back to the exploration process mentioned in Section 5.1, we could identify one more same generator cluster, comprising files exploiting CVE-2015-5122 and compressed with the lzma algorithm. These coincide with cluster flash_exploit_002 ${ }^{16}$, described in Table 4 of the ItCo report.

In a similar fashion, two classes of shared-code exploits were created: new_exp $1^{17}$ (Table 7 from ItCo report) and new_exp2 (Table 10 from ItCo report). The updated number of individuals at this point in the investigation is given in Table 3, column (iteration) 1.

\subsection{Establishing links}

The authors of the ItCo report define independent actors as the ones who "...maintain distinct infrastructure without any technical overlaps such as ip history".

Thus, we could hypothesize that two Exploitfiles would potentially share the same actor if (1) they are being distributed by the same Exploitsite or (2) their embedded PayloadFiles connect to the same C2Server ${ }^{18}$.

In order to find out about (1), a simple and direct query would be enough : Find all Exploitfiles that are distributed by the same ${ }^{19}$ Exploitserver.

On the other hand, finding about (2) would require a "join-like" operation which is better handled by graphbased technologies. After all, it would involve traversing

16. Contains one less file, not mentioned in the written report but present in the spreadsheet.

17. Contains one less file. This was expected, since it has compression value none.

18. Command-and-control $(C \& C)$ servers are used to remotely send often malicious commands to a botnet, or a compromised network of computers.Source:https://www.trendmicro.com/vinfo/us/security/definition/ command-and-control-(c-c)-server, accessed in 12/04/2017

19. We will not consider domain resolution as it is not present in the original data. However, a more precise Webserver definition, considering its assigned IP on a particular day, could also be modelled. two relationships (ExploitFile embeds PayloadFile and PayloadFile connectsTo C2Server - see Figure 1) for all Exploitfiles in the knowledge base to find out which of them share the same C2Server.

The native linking structure of the triples makes it easier to define this sparql query as depicted in Listing 4:

Listing 4: Querying for different ExploitFiles (?file1 and ?file3) with matching C2Servers (?webserver1).

select DISTINCT ?file1 where

?file1 vtinv:embeds ?file2

?file2 vtinv:connectsTo ?webserver1

?file3 vtinv:embeds ?file 4

?file4 vtinv:connectsTo ?webserver1

FILTER (?file1 != ?file3) .

This search returned 11 different ExploitFiles. If we consider the results relevant, we can transform this query into a rule for defining the new object property sameC2as, similarly to what we did in Listing 2 . Feeding this rule to the reasoner resulted in the materialized knowledge depicted in Figure 3. The yellow edges represent the recently created instances of the sameC2as property, connecting the ExploitFiles returned in the original query. These files belong to different clusters (new_HT, new_002, new_exp2 and new_exp1, derived from the materializations described in Section 5.2). In addition to listing their md5 hashes, Table 4 also informs that our conclusions regarding Exploitfiles belonging to the same actor agree with ones from the ItCo report: column ItCo table indicates the original tables comprising these files and their matching C2Server.1

\section{Rolling back}

Creating bespoke relationships allows us to promptly reuse them as necessary. For instance, we could restrict the definition of same actor by considering (1) and (2) instead of (1) or (2). The new sameC2ESas rule, building upon sameC2as, would then be:

Listing 5: Defining a more restrict definition of same actor.

vtinv:sameC2ESas (?file1, ?file3) :-

vtinv:distributes(?webserver0, ?file1),

vtinv:distributes(?webserver0, ?file3),

vtinv:sameC2as (?file1, ?file3)

Once materialised, this last rule added two instances of the object property sameC2ESas to the knowledge base, linking two distinct ExploitFiles which happen to embed the same PayloadFile. The updated counts of entities at this point in the investigation is shown in Table 3 , column (iteration) 2.

Suppose, however, that the analyst decides that the recently created relationship does not add value to the investigation. In that case, it would only be necessary to load the version prior to this materialization, and apply new queries or rules from there on.

Next, we will extend the ItCo report by enriching the original dataset, which will conveniently demonstrate the other capabilities mentioned in Table 1.

\subsection{Data enrichment}

Eventually it may be necessary to add data from other sources during the course of an investigation. There are 
TABLE 4: Correspondences between the ItCo report tables and our results in Figure 3, with regard to ExploitFiles supposedly from the same actor.

\begin{tabular}{|c|c|c|c|c|}
\hline Actor $^{20}$ & ItCo table & ExploitFile & ItCo cluster & WebServer \\
\hline APT18 & $\begin{array}{l}3 \\
6\end{array}$ & $\begin{array}{l}\text { 079a440bee0f86d8a59ebc5c4b523a07 } \\
\text { 726bd0bd6cca8d481cf6165c95528caa }\end{array}$ & $\begin{array}{l}\text { HT } \\
002\end{array}$ & 223.25 .233 .248 \\
\hline UNK1 & $\begin{array}{l}6 \\
6 \\
3\end{array}$ & $\begin{array}{l}\text { b65076f4cb6e74429dd02fcacda0bec3 } \\
\text { 8a8e9bbf1ca2a926f0a5d06217eeea55 } \\
\text { f46019f795bd721262dc69988d7e53bc }\end{array}$ & $\begin{array}{l}002 \\
002 \\
\mathrm{HT}\end{array}$ & nfitsub.com \\
\hline APT20 & $\begin{array}{l}8 \\
12\end{array}$ & $\begin{array}{l}\text { c101d289d36558c6fbe388d32bd32ab4 } \\
\text { 195bdc84f114c282e61f206dc88cd26d }\end{array}$ & $\begin{array}{l}\text { EXP1 } \\
\text { EXP2 }\end{array}$ & win7.myz.info \\
\hline DNSCalc/APT12 & $\begin{array}{l}15 \\
15\end{array}$ & $\begin{array}{l}\text { edcd313791506c623d8a2a88b9b0e84c } \\
\text { 83388058055d325a2fa5288182a41e89 }\end{array}$ & $\begin{array}{l}\text { MOVIE } \\
\text { MOVIE }\end{array}$ & $\begin{array}{l}213.186 .164 .211 \\
202.183 .129 .155\end{array}$ \\
\hline UNK10 & $\begin{array}{l}6 \\
6\end{array}$ & $\begin{array}{l}\text { 451c52652ddb28e9071078f214a327a7 } \\
\text { e33cf5b9f3991a8ee4e71f4380dd7eb1 }\end{array}$ & $\begin{array}{l}002 \\
002\end{array}$ & amxil.opmuert.org \\
\hline
\end{tabular}

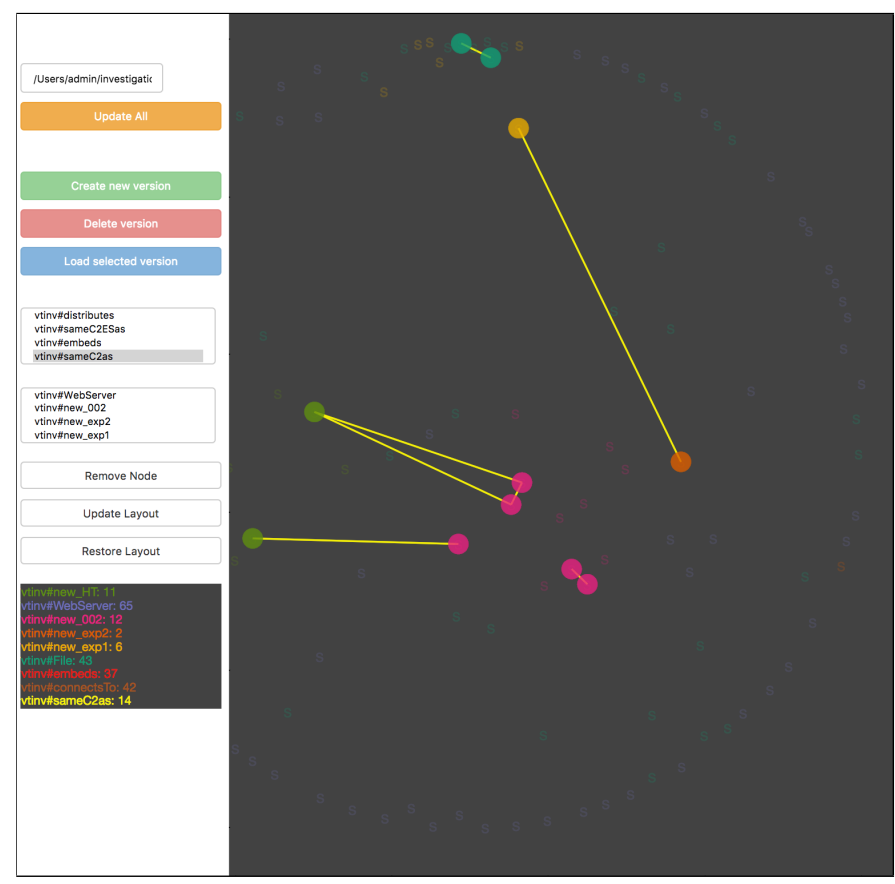

Fig. 3: Members from different clusters linked by the bespoke relationship samec2as.

two possibilities, which are not mutually exclusive: enriching data about existing entities or adding new entities

Regarding the former, we wanted to enrich the existing Payloadfiles with network data from VirusTotal. After searching this repository using their md5 hashes, it was possible to download a total of 27 file reports.

In the same way as described before, we must firstly define a mapping between our ontology and the VirusTotal network reports. For demonstration purposes, we have only mapped the object property connects To, and the datatype properties ip, domain and mo.

The resulting linked-data version comprised 20 Files (out of the initial 27) holding the relationship connectsTo with 16 WebServers. After defining the namespace "http://vt.com/", we merged it to our knowledge base and loaded it to our prototype.

As expected, the counts of File, Webserver and connectsTo have increased, as shown in Table 3, column (iteration) 3. Even though all of these "new" File instances refer to pre-existing PayloadFiles, they are still

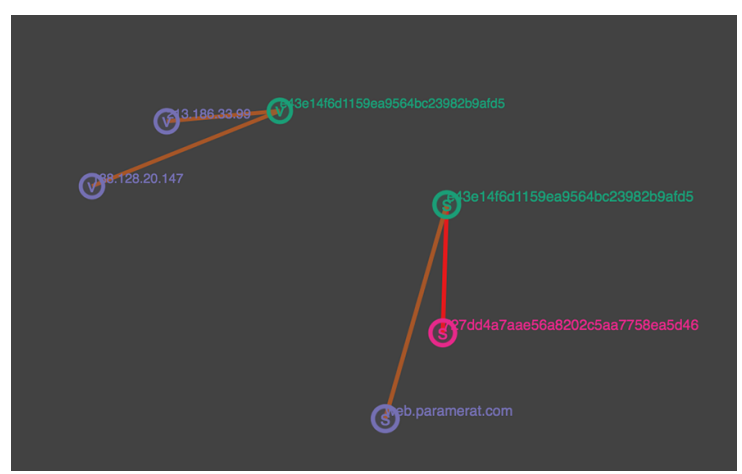

Fig. 4: Before owl:sameas: two resources (in green) referring to the same file thing.

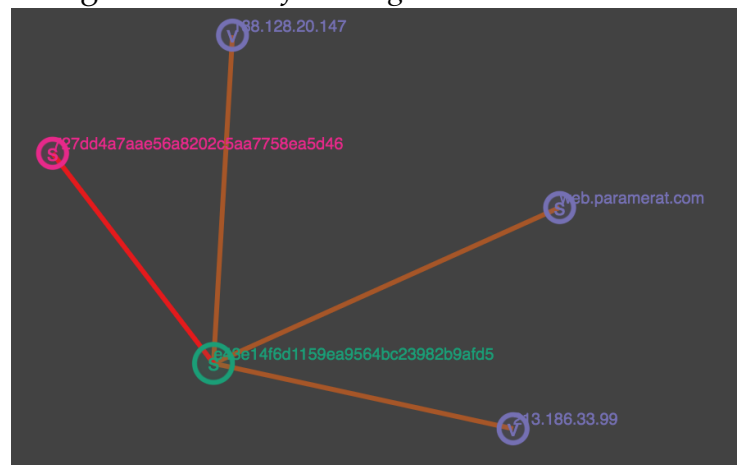

Fig. 5: After owl:sameas: integrated datatytpe properties.

distinct resources (e.g. http://ssreport.com/e43e14...b9afd5 and http://vt.com/file-e43e14...b9afd5), which might hold identical or complimentary information about the same thing: the file with md5 hash e43e14...b9afd5.

This is where the native object property owl:sameAs can be most useful: to link distinct resources referring to the same thing. Similarly to Section 5.3, we can use it to connect any two files with matching md5 hash values (Figure 4$)^{21}$. The semantic consequence is that both resources will now share the conjunction of their respective object and datatype properties (Figure 5).

In our prototype, any two individuals linked by the owl:sameas property are graphically represented as one bigger circle. This feature was implemented to reduce

21. Please refer to Figure 3 for the color legend. 
the visualization clutter, since both individuals still exist within their own namespaces (http://ssreport.com and $h t t p: / / v t . c o m$, indicated in Figures 4 and 5 by the letters 's' and ' $v$ ' ).

Column (iteration) 4 of Table 3 shows that, after the materialization of the owl:sameas property, the quantity of files decreased to the same number as iteration 2 (i.e. before the addition of the network reports in iteration 3 ). This was expected, since we have only downloaded reports for files pre-existing in the knowledge base.

For demonstration purposes, we have also established the owl:sameas property for any two WebServers with matching domain or ip. Differently from Files, the difference between versions 2 and 4 indicates that there are six new Webservers in the knowledge base, out of the sixteen added in iteration 3.

\subsection{Validation}

So far, we have demonstrated how the semantic capabilities mentioned in Table 1 could facilitate data analysis and hypothesis-testing during the course of a malware campaign investigation.

Because all the steps taken were defined and saved as rules, it is possible to submit them together with the initial dataset to a third party for auditing purposes (e.g. to check whether the results of the current investigation are sound). In addition, it also allows other analysts to extend or tune them, in order to get a different view of the same dataset. We believe that such capabilities are compatible with the reported need for "producing and sharing intelligence instead of raw IOCs".

Further, following the procedure described in Section 4, we have searched VirusTotal for the tags CVE-20155119 and $C V E-2015-5122$ in April 2016. It was possible to download 155 new files, comprising both exploits and payloads.

After extracting the ActionScript classes from the Adobe Flash exploit files and calculating their JSS, the data was converted to linked format and merged into the knowledge base. Column (iteration) 5 of Table 3 gives the new individuals count, and column (iteration) 6 reflects the counts resulting after applying the owl: sameas property to both Files and WebServers.

Then, we quickly reapplied the rules defining the four clusters and the relationship sameC2as. After the last materialisation (column 7 of Table 3), cluster new_HT was automatically populated with twenty new members and cluster new_EXP1 with four new ones.

Moreover, it was easy to spot three new sets of Exploitfiles holding the property samec2as among themselves, as indicated in Figure 6 . They are all members of cluster new_HT, endorsing the hypothesis in page 17 of the ItCo report: "The model of a single quartermaster developing and sharing generators would explain the identical nature of the malicious ActionScript classes in the HT_Exploit and flash_exploit_002 clusters."

\section{Conclusion}

We have demonstrated that our approach could indeed facilitate data exploration, making it flexible and fast

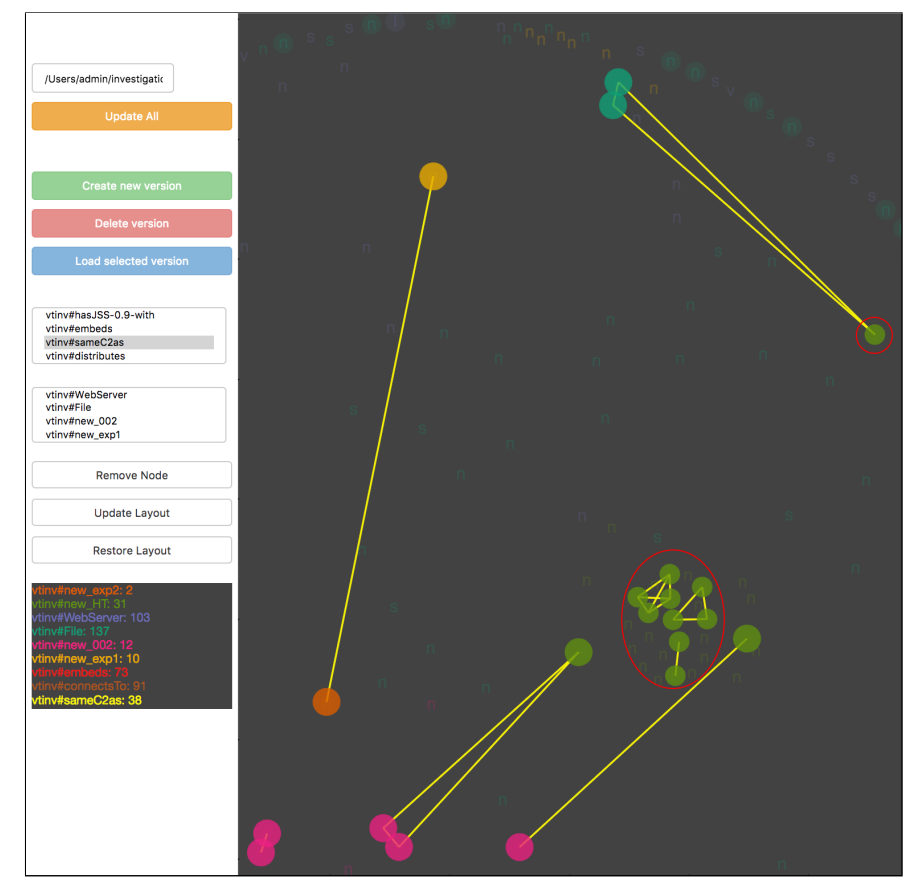

Fig. 6: The result of iteration 7 revealed ten new Exploiffiles holding the relationship sameC2as.

enough so that analysts can focus on the analysis of the results, avoiding much of the time-consuming, error-prone work in manipulating and correlating data.

Our semantic prototype allows quick and semiautomated generation of new knowledge based on expressive rules defined by the analyst during the course of an investigation. This is our contribution towards insight-generating tools which improve the balance between human reasoning (able to perform deep analyses on few items at once) and computer processing (which can evaluate large amounts of superficial information, but struggles to identify complex patterns without prior training).

It is worth noticing that not a single line of code was necessary to perform the transformations described in the previous sections. Instead, we relied solely on native semantic technology capabilities.

Moreover, the fact that information about provenance of the individuals and the meaning of all classes and relationships are explicitly defined within the data (instead of hard-wired in the source code) facilitates the integration and exploration of distinct datasets.

\section{Limitations and future work}

We acknowledge that there is a considerable preparation step in our approach, mainly because we first need to convert the original dataset to a linked format. However, once we have produced a mapping file for a specific data source, the rest of the process can be automated.

Interestingly enough, the increasing adoption of JSONLD $^{22}$ (JSON for Linked Data) within the web community (search engines such as Google are recommending it for

22. https://www.w3.org/TR/json-ld, http://json-ld.org 
information mark-up [15] ) could motivate online data providers to publish their JSON data in JSON-LD. After all, the compatibility between both formats means that only small modifications would be necessary to convert the former into the latter.

We believe that this fact, in addition to the active research regarding the scalability of current reasoners and benchmarks for linked data processing [11] could foster the development of novel semantic approaches to the exploration of the available data sources on the web.

Finally, we foresee as future work:

- Improving the prototype capabilities for malware campaign investigation by modelling domain resolution and handling dates;

- Enhancing the user interface according to visual analytics principles. The current visualisation serves solely to demonstrate the features of our semantic approach;

- Testing the prototype with expert users, to assess trade-offs such as expressiveness against semantic integrity.

\section{ACKNOWLEDGEMENTS}

We would like to thank VirusTotal for providing access to the binaries. This project is being developed during the first author's DPhil programme, which is funded by CAPES-CSF (award 9084/13-4) and supported by the Brazilian Federal Police.

\section{REFERENCES}

[1] APWG, "Phishing activity trends report - 2nd quarter 2016." [Online]. Available: http://docs.apwg.org/reports/apwg_trends_ report_q2_2016.pdf

[2] F. Merces, "The brazilian underground market." [Online]. Available: https://www.trendmicro.de/cloudcontent/us/pdfs/security-intelligence/white-papers/wp-thebrazilian-underground-market.pdf

[3] Europol. Avalanche network dismantled in international cyber operation. [Online]. Available: https://www.europol.europa.eu/ publications-documents/operation-avalanche-infographic

[4] S. L. Garfinkel, "Digital forensics research: The next 10 years," vol. 7, pp. S64-S73. [Online]. Available: http://linkinghub. elsevier.com/retrieve/pii/S1742287610000368

[5] T. Berners-Lee, J. Hendler, O. Lassila, and others, "The semantic web," vol. 284, no. 5, pp. 28-37. [Online]. Available: http://ldc.usb.ve/ yudith/docencia/UCV/ScientificAmerican_ FeatureArticle_TheSemanticWeb_May2001.pdf

[6] N. Shadbolt, T. Berners-Lee, and W. Hall, "The semantic web revisited," vol. 21, no. 3, pp. 96-101. [Online]. Available: http:/ /ieeexplore.ieee.org/xpls/abs_all.jsp?arnumber $=1637364$

[7] S. Caltagirone, A. Pendergast, and C. Betz, "The diamond model of intrusion analysis." [Online]. Available: http://oai.dtic.mil/oai/oai?verb=getRecord\& metadataPrefix $=$ html\&identifier $=$ ADA586960

[8] C. Sauerwein, C. Sillaber, A. Mussmann, and R. Breu, "Threat intelligence sharing platforms: An exploratory study of software vendors and research perspectives," in Towards Thought Leadership in Digital Transformation: 13. Internationale Tagung Wirtschaftsinformatik, WI 2017, St.Gallen, Switzerland, February 1215, 2017. [Online]. Available: https://wi2017.blob.core.windows. net/website/download/papers/WI2017-0188.pdf

[9] N. Marie and F. Gandon, "Survey of linked data based exploration systems," in Proceedings of the 3rd International Conference on Intelligent Exploration of Semantic Data-Volume 1279. CEUR-WS. org, pp. 66-77. [Online]. Available: http: $/ /$ dl.acm.org $/$ citation.cfm?id=2877806
[10] P. Szekely, C. A. Knoblock, J. Slepicka, A. Philpot, A. Singh, C. Yin, D. Kapoor, P. Natarajan, D. Marcu, K. Knight, and others, "Building and using a knowledge graph to combat human trafficking," in International Semantic Web Conference. Springer, pp. 205-221. [Online]. Available: http: //link.springer.com/chapter/10.1007/978-3-319-25010-6_12

[11] LDBC. Graph data management. [Online]. Available: http: //ldbcouncil.org/public/why-graph

[12] Y. Nenov, R. Piro, B. Motik, I. Horrocks, Z. Wu, and J. Banerjee, "RDFox: A highly-scalable RDF store." [Online]. Available: http://link.springer.com/chapter/10.1007/978-3-319-25010-6_1

[13] Ben Koehl and Ned Moran. The italian connection: An analysis of exploit supply chains and digital quartermasters. [Online]. Available: http://blog.shadowserver.org/2015/08/10/theitalian-connection-an-analysis-of-exploit-supply-chains-anddigital-quartermasters /

[14] R. Carvalho, M. Goldsmith, and S. Creese, "Applying semantic technologies to fight online banking fraud." IEEE, pp. 6168. [Online]. Available: http://ieeexplore.ieee.org/document/ $7379724 /$

[15] Google. Introduction to structured data. [Online]. Available: https://developers.google.com/search/docs/guides/introstructured-data 